ORIGINAL ARTICLE

\title{
Quorum sensing-regulated chitin metabolism provides grazing resistance to Vibrio cholerae biofilms
}

\author{
Shuyang Sun ${ }^{1,2}$, Qi Xiang Martin Tay $^{2}$, Staffan Kjelleberg ${ }^{1,2,3}$, Scott A Rice ${ }^{2,3}$ \\ and Diane McDougald ${ }^{1,2,3}$ \\ ${ }^{1}$ Centre for Marine Biofouling and Bio-Innovation, School of Biotechnology and Biomolecular Science, \\ University of New South Wales, Sydney, New South Wales, Australia; ${ }^{2}$ Singapore Centre on Environmental \\ Life Sciences Engineering, Nanyang Technological University, Singapore and ${ }^{3}$ School of Biological Sciences, \\ Nanyang Technological University, Singapore
}

\begin{abstract}
Association of Vibrio cholerae with chitinous surfaces of zooplankton is important for its persistence in marine environments, as it provides accessibility to nutrients and resistance to stresses. Predation by heterotrophic protists has a major impact on the survival of $V$. cholerae. $V$. cholerae forms biofilms as its main defensive strategy, and quorum sensing (QS) additionally regulates the production of antiprotozoal factors. The role of chitin and QS regulation in $V$. cholerae grazing resistance was investigated by exposing V. cholerae wild-type (WT) and QS mutant biofilms grown on chitin flakes to the bacteriotrophic, surface-feeding flagellate Rhynchomonas nasuta. $V$. cholerae formed more biofilm biomass on chitin flakes compared with nonchitinous surfaces. The growth of $R$. nasuta was inhibited by WT biofilms grown on chitin flakes, whereas the inhibition was attenuated in QS mutant biofilms. The chitin-dependent toxicity was also observed when the $V$. cholerae biofilms were developed under continuous flow or grown on a natural chitin source, the exoskeleton of Artemia. In addition, the antiprotozoal activity and ammonium concentration of $V$. cholerae biofilm supernatants were quantified. The ammonium levels $(3.5 \mathrm{~mm})$ detected in the supernatants of $V$. cholerae WT biofilms grown on chitin flakes were estimated to reduce the number of $R$. nasuta by $>80 \%$ in add-back experiments, and the supernatant of QS mutant biofilms was less toxic owing to a decrease in ammonium production. Transcriptomic analysis revealed that the majority of genes involved in chitin metabolism and chemotaxis were significantly downregulated in QS mutant biofilms when grown on chitin compared with the WT biofilms.
\end{abstract}

The ISME Journal (2015) 9, 1812-1820; doi:10.1038/ismej.2014.265; published online 23 January 2015

\section{Introduction}

Vibrio cholerae, the causative agent of cholera, is an opportunistic pathogen that is well adapted for survival in the marine environment during interepidemic periods (Faruque et al., 1998). Environmental surveillance for $V$. cholerae in Bangladesh revealed that $V$. cholerae persists in the aquatic environment predominately as viable but nonculturable cells in the planktonic phase and as culturable cells in biofilms formed on biotic and abiotic surfaces (Alam et al., 2006). V. cholerae biofilms associated with phytoplankton and zooplankton in water

Correspondence: D McDougald, Centre for Marine Biofouling and Bio-Innovation, School of Biotechnology and Biomolecular Sciences, University of New South Wales, Sydney, 2052, New South Wales, Australia.

E-mail: d.mcdougald@unsw.edu.au

Received 29 September 2014; accepted 8 December 2014; published online 23 January 2015 columns are suggested to be important for cholera transmission, based on the reported correlation between cholera disease outbreaks and seasonal aquatic plankton blooms (Huq et al., 1984, 1995). In addition, it has been shown that simple filtration of water through sari cloth removes up to $99 \%$ of $V$. cholerae cells by removing the zooplanktonassociated V. cholerae (Huq et al., 1996), and that this practice reduces cholera infections by $48 \%$ (Colwell et al., 2003).

Chitin is the main component of the cell walls of fungi and the exoskeletons of crustaceans and insects. Crustaceans are the majority of zooplankton, with krill and copepods comprising the largest animal biomass in the marine environment (Murphy et al., 2007). Chitin is probably the most abundant biopolymer in the marine environment, and chitin utilisation is important for $\mathrm{C}$ and $\mathrm{N}$ recycling. The chitinolytic bacteria, especially surface-associated bacteria that have higher metabolic efficiency, are essential in this process (Yu et al., 1991). 
The colonisation of chitinous surfaces of zooplankton is important for the persistence of $V$. cholerae in the aquatic environment (Huq et al., 1983; Pruzzo et al., 2008), where V. cholerae has been shown to utilise chitin as a sole nutrient source, allowing for the maintenance of metabolic activity for up to 6 months (Nahar et al., 2011). $V$. cholerae has several mechanisms that facilitate chitin colonisation, including the mannosesensitive haemagglutinin (Chiavelli et al., 2001; Meibom et al., 2004), chitin-regulated pilus (Meibom et al., 2004) and two chitin-binding proteins (36 and $53 \mathrm{kDa}$ ) (Tarsi and Pruzzo, 1999; Kirn et al., 2005; Zampini et al., 2005). The toxin coregulated pilus has also been shown to mediate biofilm differentiation on chitin surfaces (Reguera and Kolter, 2005). The chitin utilisation pathway is conserved in the Vibrionaceae and many of these genes are upregulated upon attachment of $V$. cholerae to chitin (Meibom et al., 2004; Hunt et al., 2008).

In addition to nutrient accessibility, colonisation of chitinous zooplankton has also been shown to increase the resistance of $V$. cholerae to low $\mathrm{pH}$ (Nalin et al., 1979) and alum/chlorine treatment (Chowdhury et al., 1997). Furthermore, association with chitin surfaces promotes horizontal gene transfer by inducing natural competence (Meibom et al., 2005), allowing $V$. cholerae to acquire the genes useful for adaption to various stresses both in vivo and in vitro (Bartlett and Azam, 2005).

Protozoan grazing has been identified as one of the major mortality factors faced by bacteria in the environment, and predation has a strong top-down control on $V$. cholerae populations (Worden et al., 2006). In response to protozoan grazing, $V$. cholerae has been shown to exhibit an increase in biofilm formation, where $V$. cholerae biofilms are protective against grazing pressure, whereas the planktonic counterparts are more readily consumed (Matz et al., 2005). The high cell density within biofilms also allows for the quorum sensing (QS) regulated production and accumulation of antiprotozoal factors (Sun et al., 2013). QS has also been reported to control the expression of an extracellular protease PrtV (Vaitkevicius et al., 2006) and an inhibitory factor, VasX, secreted by type VI secretion system (Ishikawa et al., 2009; Miyata et al., 2011), which are important for resistance to predation.

In this study, we investigated the role of chitin in $V$. cholerae grazing resistance by exposing $V$. cholerae biofilms grown on chitin flakes and crustacean surfaces to the bacteriotrophic surfacefeeding flagellate, Rhynchomonas nasuta. Results show that biofilms on chitinous surfaces were more resistant to predation than those on abiotic surfaces. Furthermore, we demonstrated that chitin metabolism was QS-regulated and that the ammonium produced as a by-product of chitin metabolism is toxic to $R$. nasuta.

\section{Materials and methods}

\section{Strains and culture conditions}

V. cholerae A1552 wild type (WT) and its isogenic mutant $\Delta$ hapR, a master response regulator of QS (Yildiz et al., 2004), were routinely maintained in Luria-Bertani broth and on agar plates. R. nasuta, a surface-feeding flagellate, was isolated from the Sydney Institute of Marine Science (Erken et al., 2011) and routinely grown on heat-killed Pseudomonas aeruginosa PAO1 (final concentration $10^{7}$ cells $\mathrm{ml}^{-1}$ ) in $50 \%$ nine-salt solution (NSS) medium (NSS is an artificial seawater medium that contains $17.6 \mathrm{~g} \mathrm{NaCl}, 1.47 \mathrm{~g} \mathrm{Na}_{2} \mathrm{SO}_{4}, 0.08 \mathrm{~g} \mathrm{NaHCO}_{3}, 0.25 \mathrm{~g}$ $\mathrm{KCl}, \quad 0.04 \mathrm{~g} \quad \mathrm{KBr}, \quad 1.87 \mathrm{~g} \quad \mathrm{MgCl}_{2} \bullet 6 \mathrm{H}_{2} \mathrm{O}, \quad 0.45 \mathrm{~g}$ $\mathrm{CaCl}_{2} \bullet 2 \mathrm{H}_{2} \mathrm{O}$, $0.01 \mathrm{~g} \mathrm{SrCl}_{2} \bullet 6 \mathrm{H}_{2} \mathrm{O}$ and $0.01 \mathrm{~g}_{3} \mathrm{BO}_{3}$ in $1 \mathrm{l}$ of distilled water; $50 \%$ NSS contains half of the salts of NSS medium) (Vaatanen, 1976) grown statically at room temperature (RT). Ampicillin $\left(200 \mu \mathrm{g} \mathrm{ml}^{-1}\right)$ and gentamycin $\left(200 \mu \mathrm{g} \mathrm{ml}^{-1}\right)$ were added to eradicate indigenous bacteria. $R$. nasuta was subcultured in antibiotic-free medium 3 days prior to grazing experiments.

Artemia cysts from the Great Salt Lake, UT, USA (INVE Aquaculture, Dendermonde, Belgium) were decapsulated and hatched as previously described with modification (Sung et al., 2008). Briefly, decapsulated Artemia cysts were incubated in 50\% NSS at $26^{\circ} \mathrm{C}$ for $18 \mathrm{~h}$ for hatching. The axenic Artemia were killed at the Instar I stage by freezing at $-20{ }^{\circ} \mathrm{C}$ overnight. To rule out bacterial contamination in grazing experiments, aliquots from cultures of $R$. nasuta and Artemia were plated on Luria-Bertani agar.

\section{Microtitre plate grazing assay}

To compare the grazing resistance of $V$. cholerae biofilms formed on abiotic and chitinous surfaces, batch experiments were performed in 24-well tissue culture plates as previously reported with modification (Weitere et al., 2005). V. cholerae overnight cultures were co-incubated at a final concentration of $10^{6}$ cells ml ${ }^{-1}$ with $10^{4}$ cells ml ${ }^{-1}$ of $R$. nasuta in $1 \mathrm{ml}$ of $50 \%$ NSS medium. Chitin flakes $(2 \% \mathrm{w} / \mathrm{v}$, C9213, Sigma-Aldrich, St Louis, MO, USA) or glucose $(0.01 \% \mathrm{w} / \mathrm{v})$ or both were supplemented as carbon sources. The microtitre plates were incubated at RT with shaking at 60 r.p.m. for 3 days. After incubation, $R$. nasuta was enumerated by direct inspection with an inverted light microscope $(\times 200$ magnification, CKX41, Olympus, Tokyo, Japan). V. cholerae biofilm biomass was determined by MTT staining as per manufacturer's recommendation (Cell Proliferation Kit I, Roche, Basel, Switzerland). The experiments were run in replicates of four and repeated twice.

Flow cell grazing assay

$V$. cholerae A1552 and R. nasuta were incubated in continuous flow cells $(1 \times 4 \times 40 \mathrm{~mm})$, assembled 
and prepared as previously described (Moller et al., 1998). One millilitre of $50 \%$ NSS containing $10^{6}$ cells ml ${ }^{-1}$ of $V$. cholerae A1552 and $10^{4}$ cells ml $^{-1}$ of $R$. nasuta was injected into the cell and allowed to settle for $2 \mathrm{~h}$ before the flow was restored by pumping $50 \%$ NSS into the cell at a rate of $3 \mathrm{ml} \mathrm{h}^{-1}$. Chitin flakes were glued to the bottom of flow cells using glass silicon, and glucose $(0.01 \% \mathrm{w} / \mathrm{v})$ was supplemented in the medium flow. The flow cells were incubated at RT for 3 days. $R$. nasuta was enumerated daily by inverted microscopy, and the effluents were collected and plated to determine the $V$. cholerae colony-forming units at the end of the experiment. The experiments were run in replicates of three and repeated twice.

\section{V. cholerae biofilms formed on chitinous surfaces of} zooplankton and exposed to predation

To compare biofilms on chitin flakes with those formed on natural chitinous surfaces, for example, exoskeletons of Artemia, the microtitre plate assay was modified to include $150 \mathrm{ml}^{-1}$ killed Artemia instead of chitin flakes. Owing to the difficulties encountered in quantifying the $V$. cholerae biofilm biomass formed on Artemia surfaces, only R. nasuta was enumerated microscopically with a haemocytometer. The experiments were run in replicates of four and repeated twice.

\section{Supernatant toxicity assay}

The cell-free supernatants of $V$. cholerae biofilms from the above assays were collected, filtered through $0.22 \mu \mathrm{m}$ filters (Millex-GP, Millipore, Billerica, MA, USA) and stored at $-20^{\circ} \mathrm{C}$. The antiprotozoal activity of collected supernatants was tested as previously described (Matz et al., 2005). R. nasuta (at a final concentration $10^{4}$ cells $\mathrm{ml}^{-1}$ ) was added to biofilm supernatants supplemented with heat-killed $P$. aeruginosa $\mathrm{PAO} 1$ in 24-well tissue culture plates and incubated at RT with shaking at 60 r.p.m. for 3 days. $R$. nasuta was enumerated microscopically. The experiments were run in replicates of four and repeated twice. The ammonium concentration of supernatants was determined by using the Ammonia Kit (TNT832, Hach, Lakewood, CO, USA) as per manufacturer's recommendation.

\section{Transcriptomic analysis}

V. cholerae A1552 WT and $\Delta$ hapR were grown in $50 \%$ NSS supplemented with $2 \% \mathrm{w} / \mathrm{v}$ chitin flakes at RT with shaking at 60 r.p.m. for 3 days. After removing the planktonic phase, the total RNA was extracted from biofilms formed on chitin flakes using the RNeasy Protect Bacteria Mini Kit (Qiagen, Hilden, Germany) as per the manufacturer's recommendation, and paired-end sequencing was performed on the Illumina Hi-Seq 2000 platform (San Diego, CA, USA) with 237 million reads of $100 \mathrm{bp}$ length.
Reads from Illumina were processed with Cutadapt (version 1.3) (Martin, 2011) with a quality trimming threshold of 20 and a minimum read length of $50 \mathrm{bp}$. The trimmed reads were subsequently depleted of ribosomal RNA with SortMeRNA version 1.8 (Koylova and Touzete, 2012), and the nonribosomal RNA reads mapped to the genome of $V$. cholerae (RefSeq accession numbers NC 002505 and NC 002506) using Bowtie version 2.1.0 (Langmead et al., 2009). The transcript abundance was estimated with Cufflinks version 2.1.0 (Langmead et al., 2009) using the default geometric method. Calculations of the log2 fold changes and false discovery rates were performed with Cuffdiff (Langmead et al., 2009). The differentially expressed transcripts were determined using the selection criteria of more than twofold change and $q$-value $<0.05$.

\section{Results}

Grazing resistance of V. cholerae biofilms grown on chitin flakes in batch culture

Owing to the importance of chitin utilisation and resistance to protozoan grazing for the survival of $V$. cholerae in the environment, the grazing resistance of chitin-associated $V$. cholerae and the role of QS in this process were assessed. Hence, biofilms of $V$. cholerae WT and $\Delta$ hapR strains were grown on chitin flakes and abiotic control surfaces, and exposed to predation by R. nasuta.

In the absence of a carbon source, the biofilms of $V$. cholerae formed on the surface of microtitre plates were not extensive. In the presence of glucose, there was a slight, but not significant, increase in biofilm biomass $(228 \%, P>0.05)$, whereas the biomass of biofilms formed on chitin flakes was significantly greater $(3053 \%, P<0.001)$ (Figure 1). There was no significant removal of biofilm biomass by $R$. nasuta under any of the above batch biofilms and no significant difference in biofilm biomass of $V$. cholerae WT and $\Delta$ hapR strains (Figure 1).

The antiprotozoal activity of $V$. cholerae biofilms was determined by enumeration of $R$. nasuta. In $V$. cholerae-free controls, the number of $R$. nasuta increased with the supplementation of glucose and/ or chitin flakes. The presence of $V$. cholerae WT biofilms did not significantly affect the number of $R$. nasuta on abiotic surfaces, but $R$. nasuta was killed by WT biofilms grown on chitin flakes $(P<0.001)$. In contrast, the $\Delta$ hapR biofilms supported significantly more $R$. nasuta individuals on abiotic surfaces compared with the controls and the WT biofilms $(P<0.001)$. There was no significant difference in the number of $R$. nasuta co-incubated with $\Delta$ hapR biofilms grown on chitin flakes compared with the controls. Compared with V. cholerae WT strain, $\Delta$ hapR biofilms were less toxic in all nutrient conditions $(P<0.001)$ (Figure 2). 

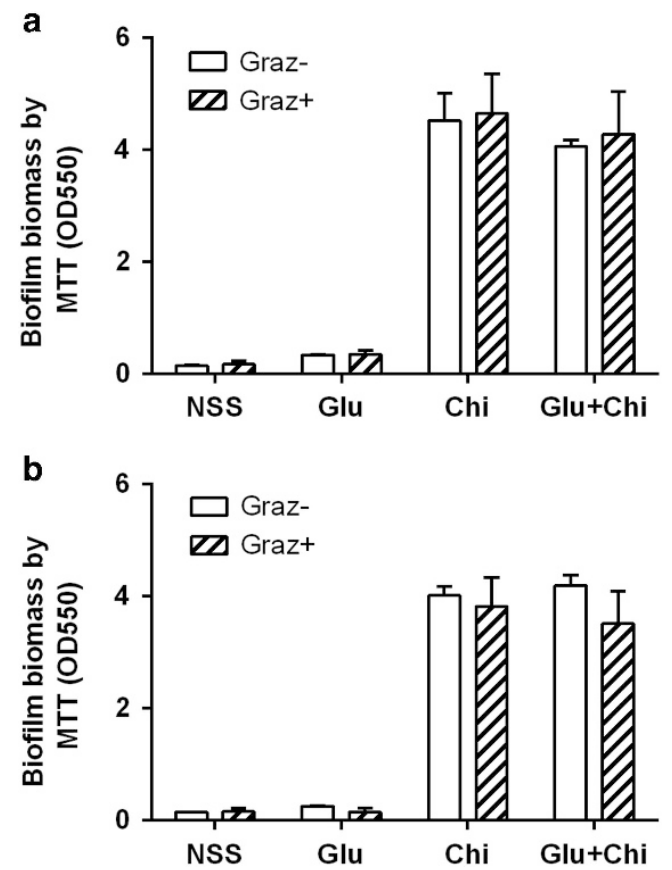

Figure 1 Grazing resistance of biofilms of $V$. cholerae grown on abiotic and chitinous surfaces. Biofilm biomass of $V$. cholerae WT (a) and $\Delta$ hapR (b) strains with no carbon source (NSS), with glucose (Glu) and/or chitin flakes (Chi) in the presence (Graz +) and absence (Graz - ) of R. nasuta.

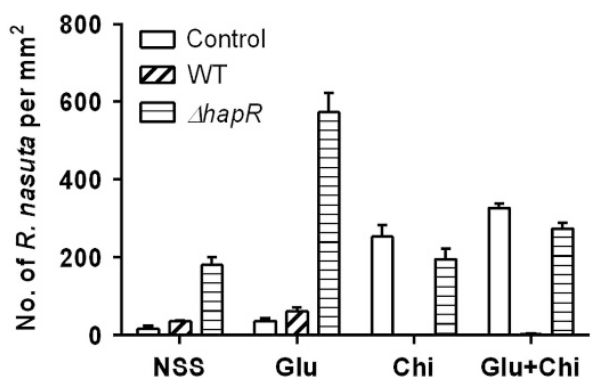

Figure 2 Effect of $V$. cholerae biofilms on survival of $R$. nasuta. Number of $R$. nasuta feeding on $V$. cholerae WT and $\Delta$ hapR biofilms with no carbon source (NSS), with glucose (Glu) and/or chitin flakes (Chi). $R$. nasuta concentrations in media without $V$. cholerae were used as controls.

Grazing resistance of $V$. cholerae biofilms grown on chitin flakes in flow cells

To determine if grazing resistance was similar under continuous flow conditions where there is dilution of extracellular components to that observed in batch cultures, $V$. cholerae WT stain and $R$. nasuta were co-incubated in flow cells with chitin or glucose. The grazing effects were determined by quantification of $V$. cholerae colony-forming units in the biofilm effluent and the numbers of $R$. nasuta.

The effluents of $V$. cholerae biofilms grown on chitin flakes contained more $V$. cholerae cells (295\%, $P<0.001$ ) than the effluents from biofilms developed in flow cells supplemented with glucose, indicating that the biofilm biomass was higher when grown on chitinous surfaces. Similar to the above grazing assays

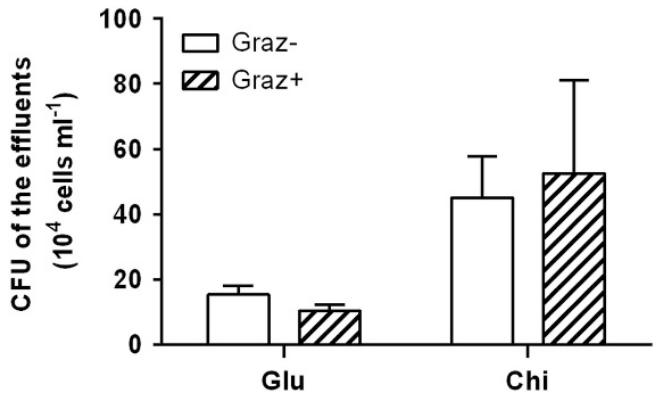

Figure 3 Biofilms formed on chitin surfaces in a flow-through system. Colony-forming units of V. cholerae WT biofilm effluents grown on glucose (Glu) and chitin flakes (Chi). Experiments were performed in flow cells in the presence $(\mathrm{Graz}+)$ and absence (Graz - ) of R. nasuta.

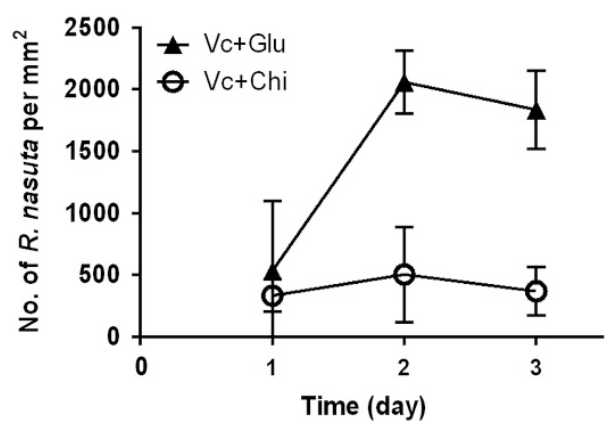

Figure 4 Effect of biofilms on survival of $R$. nasuta in a flowthrough system. Number of $R$. nasuta feeding on $V$. cholerae WT biofilms grown on glucose or chitin flakes. Experiments were performed in flow cells for 3 days and $R$. nasuta was quantified by microscopy daily.

performed in microtitre plates, predation by $R$. nasuta did not significantly reduce the biofilm biomass as the colony-forming units of effluents were similar to nongrazed controls (Figure 3).

Direct microscopic enumeration revealed that the number of $R$. nasuta increased when feeding on $V$. cholerae biofilms grown in glucose, whereas the growth of $R$. nasuta was inhibited by $V$. cholerae biofilms grown on chitin flakes, by $80 \%$ on day 3 $(P<0.001)$. In control flow cells lacking $V$. cholerae, the number of $R$. nasuta was below the detection limits, which was most likely due to nutrient deprivation (Figure 4).

\section{Grazing resistance of $V$. cholerae biofilms grown on Artemia}

In order to determine if biofilms formed on the chitinous surfaces of zooplankton were also resistant to predation, $V$. cholerae biofilms were grown first on Artemia in microtitre plates. In preliminary experiments, live Artemia fed on and depleted the $R$. nasuta population (data not shown). Thus, in the following experiments, Artemia was killed by freezing prior to addition of $V$. cholerae WT in batch cultures and the number of $R$. nasuta grazing on $V$. cholerae biofilms was compared with media controls. 
In the absence of $V$. cholerae, the number of $R$. nasuta increased more in wells containing Artemia $(1710 \%)$ than in those containing glucose $(224 \%)$, indicating that $R$. nasuta benefited from the nutrients released by freeze-killed Artemia $(P<0.001)$. The $V$. cholerae biofilms grown on abiotic surfaces did not affect the number of $R$. nasuta significantly, whereas $V$. cholerae biofilms formed on Artemia inhibited $R$. nasuta $(P<0.001)$ (Figure 5).

Ammonium produced by $V$. cholerae during chitin metabolism is toxic to $R$. nasuta

Supernatants of $V$. cholerae biofilms were collected from all the above assays and tested for their inhibitory effects on $R$. nasuta by comparing with the corresponding media controls. The supernatants of $V$. cholerae WT biofilms grown in 50\% NSS medium were nontoxic to $R$. nasuta, whereas supernatants from WT biofilms grown in glucose inhibited $R$. nasuta by $50 \%(P<0.001)$ (Figure 6$)$.

The supernatants of WT biofilms grown on chitin flakes were significantly more toxic to $R$. nasuta than those grown in glucose and media controls, resulting in a $98 \%$ decrease in $R$. nasuta numbers $(P<0.001)$. In comparison, the supernatants of $\Delta$ hapR biofilms were less toxic than the supernatants of WT biofilms when grown on chitin flakes $(P<0.001)$. Interestingly, all of the supernatants

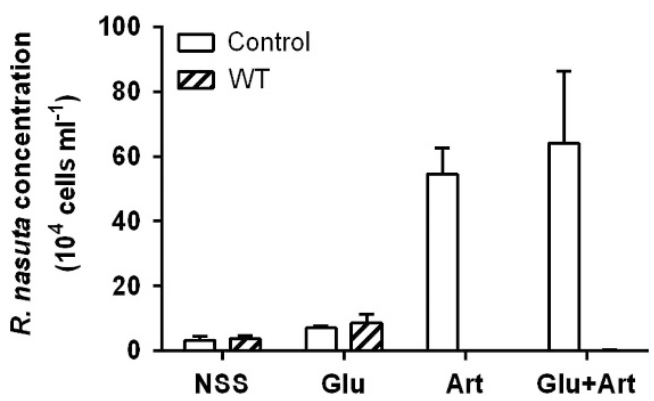

Figure 5 Grazing resistance of biofilms of $V$. cholerae formed on Artemia. Numbers of $R$. nasuta in media controls and feeding on $V$. cholerae WT biofilms with no carbon source (NSS) or with glucose (Glu) and/or Artemia (Art).

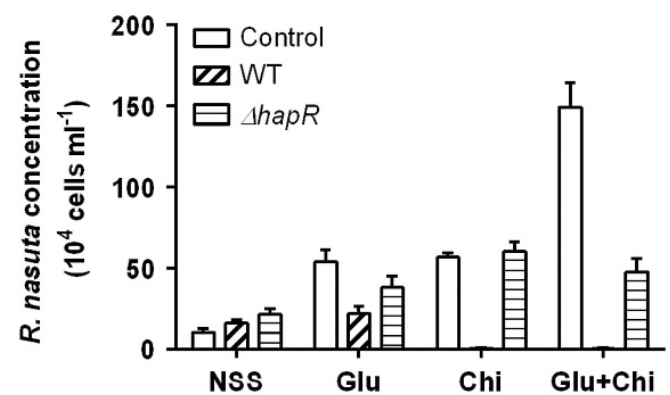

Figure 6 Toxicity of supernatants from $V$. cholerae biofilms to $R$. nasuta. Toxicity of supernatants collected from $V$. cholerae WT and $\Delta$ hapR biofilms grown in artificial seawater (NSS), glucose (Glu) and/or on chitin flakes (Chi). R. nasuta concentrations in media without $V$. cholerae were used as controls. collected from $V$. cholerae biofilms grown in flow cells and on the surfaces of Artemia did not show significant inhibition (data not shown), which was probably owing to high dilution in the flow cells and/or low chitin abundance in both conditions as discussed below.

Chitin is a modified derivative of glucose containing nitrogen, and the metabolism of chitin produces ammonia as a by-product. Therefore, the ammonium concentration of supernatants collected from $V$. cholerae biofilms grown in batch cultures was measured (Figure 7). Ammonium was not detected in media controls and was low in nonchitin supernatants. In contrast, chitin utilisation by $V$. cholerae generated a significant amount of ammonium (3.5 mM) in the supernatants $(P<0.001)$, whereas the $\Delta$ hapR biofilms produced $55 \%$ less than the WT $(P<0.001)$. The ammonium concentration of supernatants collected from $V$. cholerae biofilms grown in flow cells and on the surface of Artemia was below the detection limits (data not shown). To confirm that ammonium is toxic to $R$. nasuta, a doseresponse curve was plotted using $\mathrm{NH}_{4} \mathrm{Cl}$ in $50 \%$ NSS medium. The number of $R$. nasuta decreased with an increase in ammonium (Figure 8).

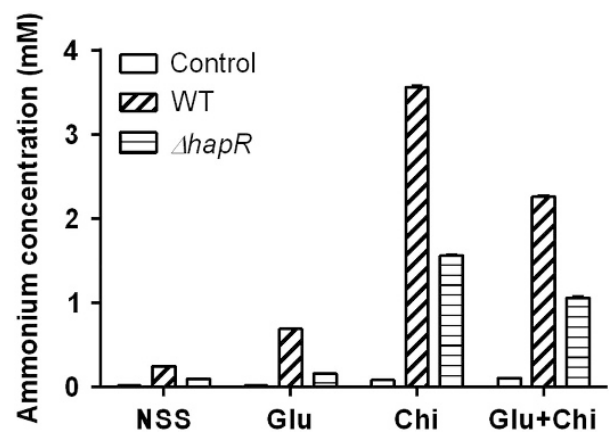

Figure 7 Ammonium concentration of $V$. cholerae biofilm supernatants. Ammonium concentration of supernatants collected from $V$. cholerae WT and $\Delta$ hapR biofilms grown in artificial seawater (NSS), glucose (Glu) and/or on chitin flakes (Chi). Ammonium concentrations of $V$. cholerae-free media was measured as controls.

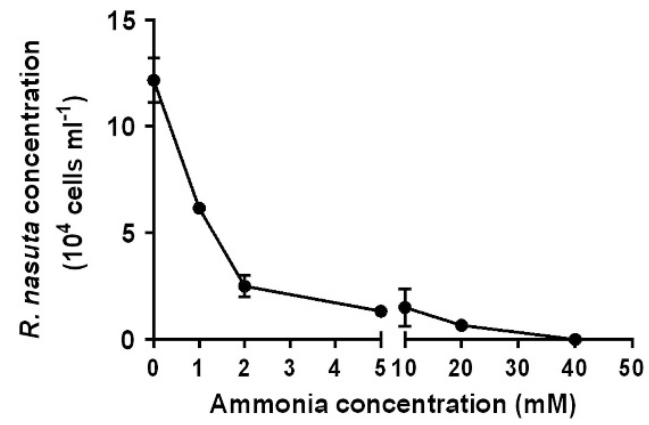

Figure 8 Effect of ammonium on survival of $R$. nasuta. Effect of increasing concentration of ammonium on $R$. nasuta survival. $R$. nasuta was quantified by microscopy after 3 days incubation in NSS medium supplemented with $\mathrm{NH}_{4} \mathrm{Cl}$. 
$Q S$ regulation of $V$. cholerae chitin utilisation

As the $V$. cholerae $\Delta$ hapR strain was less toxic to $R$. nasuta than the WT strain and produced less ammonium when grown on chitin flakes, the role of QS regulation of chitin metabolism was investigated by transcriptomic analysis of $V$. cholerae WT and $\Delta$ hapR biofilms grown on chitin flakes in batch cultures. Results demonstrated that 367 transcripts were differentially expressed with more than a twofold change (Supplementary Table S1).

The chitinolytic pathway of $V$. cholerae (Hunt et al., 2008) begins with the degradation of chitin by chitinase and the transport of $N$-acetylglucosamine (GlcNAc) monomers or dimers into the periplasm. The transcripts of genes that are involved in this process were largely unchanged in the $\Delta h a p R$ biofilms compared with the WT biofilms grown on chitin, with the exception of two chitinases (VC0769 and VCA0027), which were expressed at a higher level in $\Delta$ hapR biofilms. Following the transport of GlcNAc monomers and dimers, the products of degradation are delivered into central metabolism as fructose-6-phosphate, acetate and ammonium. Among the 22 genes involved in GlcNAc catabolism, transcripts of 19 genes, including the entire chitin catabolic operon (VC0611-VC0620), were expressed at a significantly lower level in the QS-negative $\Delta h a p R$ biofilms, whereas the transcripts of the remaining 3 genes were not significantly different (Table 1). In addition, transcripts of the genes coding for chitinregulated pilus (VC2423, VC2424 and VC1612) and the majority of the methyl-accepting chemotaxis genes (29 of total 45 MCPs) were repressed in the $\Delta$ hapR biofilms, whereas only 2 MCP genes were upregulated. The RNA-Seq data also confirmed QS regulation of previously reported genes, including those involved in Vibrio polysaccharide production, flagella synthesis, virulence, natural competence, type VI secretion system and c-di-GMP synthesis/ degradation (Supplementary Table S1).

\section{Discussion}

V. cholerae biofilms formed on chitin surfaces are grazing resistant

Results show that $V$. cholerae forms significantly more biofilm biomass on chitin flakes than on abiotic surfaces in both microtitre plates and continuous flow cells. It has been reported that chitin colonisation and utilisation are ubiquitous among vibrios, with all completed genomes of vibrios having multiple chitinases (Grimes et al., 2009). The association with chitin is important for the long-term persistence of $V$. cholerae in the environment. $V$. cholerae can use chitin as a sole carbon source (Nahar et al., 2011), and colonisation of chitinous zooplankton has been shown to increase the resistance of $V$. cholerae to low pH (Nalin et al., 1979) and alum/chlorine treatment (Chowdhury et al., 1997). Various chitin attachment mechanisms have been identified (Meibom et al., 2004), although the role of mannose-sensitive haemagglutinin in chitin association is still under debate (Watnick et al., 1999). When the $V$. cholerae biofilms grown on chitin flakes were exposed to predation by $R$. nasuta, there was little effect of grazing on biofilm biomass and this was in line with our previous observation that biofilm formation protects $V$. cholerae from protozoan grazing (Matz et al., 2005).

Ammonium produced by $V$. cholerae chitin metabolism is toxic to $R$. nasuta

In both microtitre plates and flow cells, $V$. cholerae biofilms formed on chitin flakes were more toxic to $R$. nasuta compared with biofilms formed on abiotic

Table 1 Differentially regulated genes between $V$. cholerae WT and $\Delta$ hapR biofilms in GlcNAc catabolism

\begin{tabular}{|c|c|c|}
\hline Gene & Description & $\Delta$ hapR/WT log2 fold \\
\hline VC0611 & Phosphoglucomutase/phosphomannomutase & -1.74 \\
\hline VC0612 & Cellobiose/cellodextrin-phosphorylase & -1.92 \\
\hline VC0613 & Beta- $N$-acetylhexosaminidase & -1.54 \\
\hline VC0614 & Hypothetical protein & -1.55 \\
\hline VC0615 & Endoglucanase-like protein & -1.01 \\
\hline VC0616 & Peptide ABC transporter ATP-binding protein & -1.63 \\
\hline VC0617 & Peptide ABC transporter ATP-binding protein & -1.78 \\
\hline$V C 0618$ & Peptide ABC transporter permease & -1.42 \\
\hline VC0619 & Peptide ABC transporter permease & -1.96 \\
\hline VC0620 & Peptide ABC transporter substrate-binding protein & -1.10 \\
\hline VC0994 & $N$-acetylglucosamine-6-phosphate deacetylase & -1.22 \\
\hline VC0995 & PTS system $N$-acetylglucosamine-specific transporter subunit IIABC & -1.48 \\
\hline VC1280 & Hypothetical protein & -1.47 \\
\hline$V C 1281$ & PTS system cellobiose-specific transporter subunit IIB & -2.29 \\
\hline$V C 1282$ & PTS system cellobiose-specific transporter subunit IIC & -2.17 \\
\hline$V C 1284$ & 6-Phospho-beta-glucosidase & -1.77 \\
\hline VC1285 & Hypothetical protein & -1.06 \\
\hline VC2217 & Beta- $N$-acetylhexosaminidase & -1.93 \\
\hline VCA1025 & Glucosamine-6-phosphate deaminase & -1.48 \\
\hline
\end{tabular}

Abbreviations: ABC, ATP-binding cassette; PTS, phosphotransferase system. 
surfaces, indicating the existence of antiprotozoal factor(s) produced by $V$. cholerae biofilms in association with chitin. Furthermore, the chitindependent antiprotozoal activity was also observed when biofilms were grown on the surface of Artemia. Supernatants collected from $V$. cholerae biofilms grown on chitin flakes in batch cultures were inhibitory to $R$. nasuta and, subsequently, ammonium was identified to be responsible for the antiprotozoal activity in the supernatant. This metabolite-based grazing resistance may be a general feature of environmental bacteria. For example, biofilms of Pseudomonas fluorescens CHA0 produce the metabolites hydrogen cyanide and 2,4diacetylphloroglucinol, which have antiprotozoal activities against bacteriotrophic protists (Jousset et al., 2006).

Ammonia/ammonium at high concentrations is generally toxic to eukaryotic cells. It has been reported that the amphipod Gammarus pulex is inhibited by $<0.1 \mathrm{~mm}$ ammonium (Berenzen et al., 2001; Prenter et al., 2004), and bacterial-derived ammonia in the intestine is largely accepted as the main source for hyperammonaemia in patients with liver disease (Sherlock, 1987). Interestingly, ammonia is also an important environmental signal in the regulation of the life cycle of Dictyostelium discoideum, where high ammonia concentrations inhibit fruiting body formation (Mahadeo and Parent, 2006). The dose-response curve of $R$. nasuta to ammonium demonstrated that $2 \mathrm{~mm}$ ammonium was lethal for $80 \%$ of $R$. nasuta individuals. As the supernatants of $V$. cholerae WT biofilms grown on chitin flakes contained $3.5 \mathrm{~mm}$ ammonium, these biofilms are even more toxic than $R$. nasuta.

$R$. nasuta was also inhibited when co-incubated with $V$. cholerae biofilms formed on chitin flakes in flow cells or on Artemia. In the marine environment, seasonal plankton blooms may provide enough chitin for $V$. cholerae metabolism to produce inhibitory amounts of ammonium (Huq et al., 1984). It has also been proposed that the inhibitory factors may accumulate to higher concentrations within biofilms than in supernatants owing to high cell density and retention of cell-derived compounds by exopolymeric substances (Matz et al., 2005). In addition, the antiprotozoal activity of $V$. cholerae biofilms in association with chitinous surfaces may also benefit from other inhibitory factors, for example, PrtV (Vaitkevicius et al., 2006), VasX (Ishikawa et al., 2009; Miyata et al., 2011 ) and the unknown factor secreted by $V$. cholerae biofilms (Matz et al., 2005).

QS regulation of chitin metabolism and antiprotozoal activity in V. cholerae biofilms

The reduced production of ammonium and decreased toxicity against $R$. nasuta of $V$. cholerae $\Delta$ hap $R$ biofilms grown on chitin flakes indicates that
QS controls V. cholerae chitin utilisation. Transcriptomic analysis revealed that 19 of the 22 genes involved in GlcNAc catabolism, as well as functionally related chitin-regulated pilus synthesis and chemotaxis, were positively regulated by QS in $V$. cholerae biofilms.

Interestingly, two putative chitinases, VC0769 and VCA0027, were downregulated by QS in V. cholerae biofilms, indicating that the five chitinases in $V$. cholerae might be redundant or functionally differentiated. The negative regulation of chitinase by QS has been reported previously, where VCA0811 was found to be repressed by QS in a microarray study of $V$. cholerae rugosity (Yildiz et al., 2004), and chitinase A of Vibrio harveyi was expressed at higher level in a QS-negative mutant strain (Defoirdt et al., 2010). One possible explanation is that the expression of chitinases may be a nutrientexploring strategy used by planktonic $V$. cholerae cells rather than in high-density biofilms. When chitin is present in the surrounding environment, the secreted chitinases degrade chitin and generate soluble oligomers of GlcNAc, which act as signals for $V$. cholerae chemotaxis, chitin attachment and utilisation (Meibom et al., 2004). To date, only VC1952 and VCA0027 have been confirmed to have catabolic activity for chitin and the double-deletion mutant could not grow on chitin as the sole carbon source (Meibom et al., 2004). Thus, it is possible that the remaining three chitinases may not have catabolic activity.

It has been proposed that many mechanisms involved in $V$. cholerae environmental persistence are important in pathogenesis as well (Vezzulli et al., 2008). Indeed, it has been reported that six genes (VC0613, VC0614, VC0616, VC0619, VC1284 and VC2424) involved in chitin utilisation are also induced during colonisation of the infant mice small intestine (Mandlik et al., 2011). Therefore, the QS-regulated chitin utilisation genes of $V$. cholerae biofilms may play a role in infection by V. cholerae.

Chitin association has been reported to provide $V$. cholerae nutrient accessibility and to induce natural competence of $V$. cholerae. The data presented here show that biofilm formation of $V$. cholerae on chitin provides increased grazing resistance and is toxic to the heterotrophic protist, $R$. nasuta, owing to the production of ammonia as a by-product of chitin metabolism. Furthermore, we show that chitin metabolism is QS-regulated. These results provide an explanation for the association of $V$. cholerae with chitinous exoskeletons of zooplankton as well as a role for QS-regulated phenotypes in persistence and survival in the marine environment.

\section{Conflict of Interest}

The authors declare no conflict of interest. 


\section{Acknowledgements}

This study was supported by the Australian Research Council (ARC) grant DP1096481, the Centre for Marine Bio-Innovation (CMB) at the University of New South Wales and the Singapore Centre on Environmental Life Sciences Engineering, Nanyang Technological University, Singapore.

\section{References}

Alam M, Sultana M, Nair GB, Sack RB, Sack DA, Siddique AK et al. (2006). Toxigenic Vibrio cholerae in the aquatic environment of Mathbaria, Bangladesh. Appl Environ Microbiol 72: 2849-2855.

Bartlett DH, Azam F. (2005). Microbiology. Chitin, cholera, and competence. Science 310: 1775-1777.

Berenzen N, Schulz R, Liess M. (2001). Effects of chronic ammonium and nitrite contamination on the macroinvertebrate community in running water microcosms. Water Res 35: 3478-3482.

Chiavelli DA, Marsh JW, Taylor RK. (2001). The mannosesensitive hemagglutinin of Vibrio cholerae promotes adherence to zooplankton. Appl Environ Microbiol 67: 3220-3225.

Chowdhury MA, Huq A, Xu B, Madeira FJ, Colwell RR. (1997). Effect of alum on free-living and copepodassociated Vibrio cholerae $\mathrm{O} 1$ and O139. Appl Environ Microbiol 63: 3323-3326.

Colwell RR, Huq A, Islam MS, Aziz KM, Yunus M, Khan NH et al. (2003). Reduction of cholera in Bangladeshi villages by simple filtration. Proc Natl Acad Sci USA 100: 1051-1055.

Defoirdt T, Darshanee Ruwandeepika HA, Karunasagar I, Boon N, Bossier P. (2010). Quorum sensing negatively regulates chitinase in Vibrio harveyi. Environ Microbiol Rep 2: 44-49.

Erken M, Weitere M, Kjelleberg S, McDougald D. (2011). In situ grazing resistance of Vibrio cholerae in the marine environment. FEMS Microbiol Ecol 76: 504-512.

Faruque SM, Albert MJ, Mekalanos JJ. (1998). Epidemiology, genetics, and ecology of toxigenic Vibrio cholerae. Microbiol Mol Biol Rev 62: 1301-1314.

Grimes DJ, Johnson CN, Dillon KS, Flowers AR, Noriea NF 3rd, Berutti T. (2009). What genomic sequence information has revealed about Vibrio ecology in the ocean-a review. Microb Ecol 58: 447-460.

Hunt DE, Gevers D, Vahora NM, Polz MF. (2008). Conservation of the chitin utilization pathway in the Vibrionaceae. Appl Environ Microbiol 74: 44-51.

Huq A, Small EB, West PA, Huq MI, Rahman R, Colwell RR. (1983). Ecological relationships between Vibrio cholerae and planktonic crustacean copepods. Appl Environ Microbiol 45: 275-283.

Huq A, West PA, Small EB, Huq MI, Colwell RR. (1984). Influence of water temperature, salinity, and $\mathrm{pH}$ on survival and growth of toxigenic Vibrio cholerae serovar 01 associated with live copepods in laboratory microcosms. Appl Environ Microbiol 48: 420-424.

Huq A, Colwell RR, Chowdhury MA, Xu B, Moniruzzaman SM, Islam MS et al. (1995). Coexistence of Vibrio cholerae O1 and O139 Bengal in plankton in Bangladesh. Lancet 345: 1249.
Huq A, Xu B, Chowdhury MA, Islam MS, Montilla R, Colwell RR. (1996). A simple filtration method to remove plankton-associated Vibrio cholerae in raw water supplies in developing countries. Appl Environ Microbiol 62: 2508-2512.

Ishikawa T, Rompikuntal PK, Lindmark B, Milton DL, Wai SN. (2009). Quorum sensing regulation of the two hcp alleles in Vibrio cholerae O1 strains. PLoS One 4: e6734.

Jousset A, Lara E, Wall LG, Valverde C. (2006). Secondary metabolites help biocontrol strain Pseudomonas fluorescens CHA0 to escape protozoan grazing. Appl Environ Microbiol 72: 7083-7090.

Kirn TJ, Jude BA, Taylor RK. (2005). A colonization factor links Vibrio cholerae environmental survival and human infection. Nature 438: 863-866.

Kopylova E, Noe L, Touzet H. (2012). SortMeRNA: Fast and accurate filtering of ribosomal RNAs in metatranscriptomic data. Bioinformatics 28: 3211-3217.

Langmead B, Trapnell C, Pop M, Salzberg S. (2009). Ultrafast and memory-efficient alignment of short DNA sequences to the human genome. Genome Biology 10: R25.

Mahadeo DC, Parent CA. (2006). Signal relay during the life cycle of Dictyostelium. Curr Top Dev Biol 73: 115-140.

Mandlik A, Livny J, Robins WP, Ritchie JM, Mekalanos JJ, Waldor MK. (2011). RNA-Seq-based monitoring of infection-linked changes in Vibrio cholerae gene expression. Cell Host Microbe 10: 165-174.

Martin M. (2011). Cutadapt removes adapter sequences from high-throughput sequencing reads. EMBnet.journal 17: 10-12.

Matz C, McDougald D, Moreno AM, Yung PY, Yildiz FH, Kjelleberg S. (2005). Biofilm formation and phenotypic variation enhance predation-driven persistence of Vibrio cholerae. Proc Natl Acad Sci USA 102: 16819-16824.

Meibom KL, Li XB, Nielsen AT, Wu CY, Roseman S, Schoolnik GK. (2004). The Vibrio cholerae chitin utilization program. Proc Natl Acad Sci USA 101: 2524-2529.

Meibom KL, Blokesch M, Dolganov NA, Wu CY, Schoolnik GK. (2005). Chitin induces natural competence in Vibrio cholerae. Science 310: 1824-1827.

Miyata ST, Kitaoka M, Brooks TM, McAuley SB, Pukatzki S. (2011). Vibrio cholerae requires the type VI secretion system virulence factor VasX to kill Dictyostelium discoideum. Infect Immun 79: 2941-2949.

Moller S, Sternberg C, Andersen JB, Christensen BB, Ramos JL, Givskov $M$ et al. (1998). In situ gene expression in mixed-culture biofilms: evidence of metabolic interactions between community members. Appl Environ Microbiol 64: 721-732.

Murphy EJ, Watkins JL, Trathan PN, Reid K, Meredith MP, Thorpe SE et al. (2007). Spatial and temporal operation of the Scotia Sea ecosystem: a review of large-scale links in a krill centred food web. Philos Trans $R$ Soc Lond B Biol Sci 362: 113-148.

Nahar S, Sultana M, Naser MN, Nair GB, Watanabe H, Ohnishi $\mathrm{M}$ et al. (2011). Role of shrimp chitin in the ecology of toxigenic Vibrio cholerae and cholera transmission. Front Microbiol 2: 260.

Nalin DR, Daya V, Reid A, Levine MM, Cisneros L. (1979). Adsorption and growth of Vibrio cholerae on chitin. Infect Immun 25: 768-770. 
Prenter J, MacNeil C, Dick JT, Riddell GE, Dunn AM. (2004). Lethal and sublethal toxicity of ammonia to native, invasive, and parasitised freshwater amphipods. Water Res 38: 2847-2850.

Pruzzo C, Vezzulli L, Colwell RR. (2008). Global impact of Vibrio cholerae interactions with chitin. Environ Microbiol 10: 1400-1410.

Reguera G, Kolter R. (2005). Virulence and the environment: a novel role for Vibrio cholerae toxincoregulated pili in biofilm formation on chitin. J Bacteriol 187: 3551-3555.

Sherlock S. (1987). Chronic portal systemic encephalopathy: update 1987. Gut 28: 1043-1048.

Sun S, Kjelleberg S, McDougald D. (2013). Relative contributions of Vibrio polysaccharide and quorum sensing to the resistance of Vibrio cholerae to predation by heterotrophic protists. PLoS One 8: e56338.

Sung YY, Pineda C, MacRae TH, Sorgeloos P, Bossier P. (2008). Exposure of gnotobiotic Artemia franciscana larvae to abiotic stress promotes heat shock protein 70 synthesis and enhances resistance to pathogenic Vibrio campbellii. Cell Stress Chaperones 13: 59-66.

Tarsi R, Pruzzo C. (1999). Role of surface proteins in Vibrio cholerae attachment to chitin. Appl Environ Microbiol 65: 1348-1351.

Vaatanen P. (1976). Microbiological studies on coastal waters of the Northern Baltic sea, I. Distribution and abundance of bacteria and yeasts in the Tvarminne area. Walter Andre Nottback Found Sci Rep 1: 1-58.

Vaitkevicius K, Lindmark B, Ou G, Song T, Toma C, Iwanaga $M$ et al. (2006). A Vibrio cholerae protease needed for killing of Caenorhabditis elegans has a role in protection from natural predator grazing. Proc Natl Acad Sci USA 103: 9280-9285.

Vezzulli L, Guzman CA, Colwell RR, Pruzzo C. (2008). Dual role colonization factors connecting Vibrio cholerae's lifestyles in human and aquatic environments open new perspectives for combating infectious diseases. Curr Opin Biotechnol 19: 254-259.
Watnick PI, Fullner KJ, Kolter R. (1999). A role for the mannose-sensitive hemagglutinin in biofilm formation by Vibrio cholerae El Tor. J Bacteriol 181: 3606-3609.

Weitere M, Bergfeld T, Rice SA, Matz C, Kjelleberg S. (2005). Grazing resistance of Pseudomonas aeruginosa biofilms depends on type of protective mechanism, developmental stage and protozoan feeding mode. Environ Microbiol 7: 1593-1601.

Worden AZ, Seidel M, Smriga S, Wick A, Malfatti F, Bartlett D et al. (2006). Trophic regulation of Vibrio cholerae in coastal marine waters. Environ Microbiol 8: 21-29.

Yildiz FH, Liu XS, Heydorn A, Schoolnik GK. (2004). Molecular analysis of rugosity in a Vibrio cholerae O1 El Tor phase variant. Mol Microbiol 53: 497-515.

$\mathrm{Yu}$ C, Lee AM, Bassler BL, Roseman S. (1991). Chitin utilization by marine bacteria. A physiological function for bacterial adhesion to immobilized carbohydrates. J Biol Chem 266: 24260-24267.

Zampini M, Pruzzo C, Bondre VP, Tarsi R, Cosmo M, Bacciaglia A et al. (2005). Vibrio cholerae persistence in aquatic environments and colonization of intestinal cells: involvement of a common adhesion mechanism. FEMS Microbiol Lett 244: 267-273.

(1) () $\odot$ This work is licensed under a Creative (c) ${ }_{\text {ay }}$ ne wo Commons Attribution-NonCommercialNoDerivs 3.0 Unported License. The images or other third party material in this article are included in the article's Creative Commons license, unless indicated otherwise in the credit line; if the material is not included under the Creative Commons license, users will need to obtain permission from the license holder to reproduce the material. To view a copy of this license, visit http://creativecommons.org/ licenses/by-nc-nd/3.0/

Supplementary Information accompanies this paper on The ISME Journal website (http://www.nature.com/ismej) 